\title{
La inteligencia artificial y la toma de decisiones gerenciales
}

Artificial intelligence and managerial decision making

Nadiuska Marelyn Diestra Quinto ${ }^{(\mathbb{D})}$ *, Alexandra Janet Cordova Villodas ${ }^{b}{ }^{\mathbb{D}}$, Caren Patricia Caruajulca Montero $^{c(\mathbb{D})}$, Damaris Laura Esquivel Cueva ${ }^{(\mathbb{D} \text {, Sheyla Alcira Nina Vera }}{ }^{\text {(i) }}$ a,b,c,d,e Universidad Nacional Mayor de San Marcos, Lima, Perú

\section{Resumen}

La toma de decisiones gerenciales se ha vuelto compleja en el mundo actual, esto debido al desarrollo acelerado de la tecnología, lo que origina que los encargados de tomar las decisiones procesen grandes cantidades de información variada de forma inmediata y se enfrenten a las posibles consecuencias y riesgos inherentes de este acelerado dinamismo, ya que la información debe recopilarse e interpretarse correctamente para poder concluir en una alternativa idónea. Ante esta problemática que viven las organizaciones, la inteligencia artificial (IA) se ha vuelto un aliado ideal en la toma de decisiones, así como un posible reemplazo del gestor de decisiones humano. Así pues, el objetivo del presente trabajo consiste en identificar la relación de la IA y la toma de decisiones organizacionales. Como metodología se empleó una revisión sistemática de la literatura que permitió obtener resultados abocados a la dependencia que existe entre la IA y la toma de decisiones gerenciales, lo que está generando una automatización acelerada de los procesos en la gestión empresarial. Esto conduce a la reflexión que, a pesar de la existencia de una fuerte competencia en el mundo empresarial, que ha llevado a las empresas a buscar medios que generen una eficacia y eficiencia inmediata sin prever las posibles consecuencias, se debe orientar a obtener una relación equilibrada de la IA y la toma de decisiones.

Palabras claves: Decisiones administrativas; inteligencia artificial; planeamiento estratégico; computación cognitiva; aprendizaje automático

\footnotetext{
Abstract

* Autor de correspondencia:

Tel.: +51-977-367-679

E-mail: nadiuska.diestra@unmsm.edu.pe

DOI: https://doi.org/10.17162/riva.v8i1.1631

Recibido: 13/09/2021 Aceptado: 21/11/2021
}

Managerial decision-making has become complex in today's world, due to the accelerated development of technology, which causes decision makers to process large amounts of varied information immediately and face the possible consequences and inherent risks of this 
accelerated dynamism. Since, the information must be collected and interpreted correctly in order to conclude an ideal alternative. Faced with this problem that organizations experience, Artificial Intelligence (Al) has become an ideal ally in decision-making, as well as a possible replacement for the human decision-maker. Thus, the objective of this work is to identify the relationship between $\mathrm{Al}$ and decision-making in organizations. In this article, a systematic review of the literature was used as a methodology that allowed obtaining results aimed at the dependence that exists between Al and managerial decision-making, which is generating an accelerated automation of processes in business management. This leads to the reflection that, although there is strong competition in the business world that has led companies to seek means that generate immediate effectiveness and efficiency without anticipating the possible consequences, it should be aimed at obtaining a balanced relationship of $\mathrm{Al}$ and decision making.

Keywords: Administrative decisions; artificial intelligence; strategic planning; cognitive computing; machine learning

\section{Introducción}

El tiempo es un elemento muy valioso, y es por ello que cada segundo de su vida debe ser bien aprovechado. Esto mismo sucede en el mundo de los negocios y se visualiza en la toma de decisiones, las cuales se deben efectuar de manera apresurada para la resolución de conflictos. No obstante, este aspecto puede afectar el resultado de la toma de decisiones (Ahituv et al., 1998), ya que estas se toman en un ambiente de incertidumbre y bajo presión, lo cual genera que empleen la heurística para sobrestimar e ignorar otras informaciones (Janis, 1991). Además, este proceso de decisión humana resulta ser muy complejo (Nietzsche, 2015), por la cantidad de alternativas aparentemente equivalentes que se disponen, generando así que se enfrenten a una variedad de posibles consecuencias y riesgos inherentes que pueden conducir a una equívoca toma de decisiones (Lyengar y Lepper, 2000).

Por otro lado, la toma de decisiones se ve influenciada por las experiencias, emociones y variaciones interpersonales, que afecta la recepción de información por parte de una persona al momento de tomar decisiones, y se refleja en resultados problemáticos (De Dreu et al., 2008), como puede ser la fatiga durante el proceso de toma de decisiones, el cual puede conducir a una mala calidad de estas mismas, pues la energía mental del individuo se agota de forma continua.

En este sentido, la inteligencia artificial puede tener un impacto significativo en la toma de decisiones, debido a que introduce un conjunto de nuevos desafíos a este viejo problema (Von Krogh, 2018). Por ello, el uso de la IA en la toma de decisiones organizacionales sigue 
siendo una de las principales aplicaciones (Cao et al., 2021), dado que crea nueva información y predicciones a partir de grandes conjuntos de datos para la resolución de problemas, especialmente de algoritmos de aprendizaje automático (Von Krogh, 2018). Según un informe reciente del MIT Sloan Management Review y Boston Consulting Group, el $57 \%$ de las empresas encuestadas prueba la IA y el 59 \% poseen una estrategia de IA (Ransbotham et al., 2020). Esto genera nuevas áreas de aplicación, como cambiar la forma y perspectiva en que las organizaciones toman decisiones (Aaldering y Song, 2020).

La implementación de la IA en las organizaciones o empresas es fundamental para tomar eficientes decisiones en esta área. Un beneficio de la implementación de la inteligencia artificial en las empresas es que esta puede ayudar a los gerentes a detectar anomalías, al proporcionar información en tiempo real sobre señales de advertencia temprana de problemas, lo cual permite la posibilidad de acciones correctivas oportunas (Jarrahi, 2018).

De acuerdo con lo mencionado, la presente investigación tiene como objetivo analizar la relación de la inteligencia artificial y la toma de decisiones en el mundo de los negocios. Asimismo, se busca identificar la influencia que conlleva esta interrelación, con el fin de aportar una revisión teórica de estos conceptos y establecer un comentario crítico basado en la información revisada.

Es así que la investigación es descriptiva y de revisión bibliográfica. Se apoya en artículos científicos de la base de datos Scopus, los cuales han pasado por un proceso de análisis y estudio profundo con el fin de extraer los elementos de interés que servirán en la investigación. De igual manera, se ha priorizado la utilización de artículos de enfoque cualitativo, por lo que el análisis principal de este trabajo se centra en el constructo teórico de la IA y la toma de decisiones.

El artículo está organizado en las siguientes partes: en primer lugar, se realizará una revisión de la literatura sobre la definición de la IA y la toma de decisiones, sus respectivas aplicaciones en las empresas, así como la relación que existe entre la toma de decisiones con la IA en el tiempo. En segundo lugar, se describe la metodología utilizada para la elaboración del mismo. Posteriormente, se presentarán los resultados y discusiones que incluye la elaboración de un comentario crítico que se contrastará con los hallazgos de la investigación acerca de la IA y la toma de decisiones. Finalmente, se presentarán las conclusiones de la presente investigación. 


\section{Desarrollo o revisión}

\subsection{La inteligencia artificial y algunas aplicaciones}

Actualmente existen diversos conceptos que pueden ayudar a definir la inteligencia artificial, muchos de ellos mencionan que son sistemas inteligentes asociados a la mente humana capaces de razonar y aprender (Russell et al., 1995), lo que les permite interactuar y resolver problemas (Nilsson, 1971). La IA incluye un grupo variado de herramientas, métodos y algoritmos (Jarrahi, 2018), que están direccionados, según algunos autores, a complementar y aumentar las habilidades humanas, en vez de reducirlas o limitarlas (Raisch y Krakowski, 2020).

De igual forma, durante estos últimos años, el término «inteligencia artificial» ha resurgido volviéndose en «trending topic» (Burggräf et al., 2020), es decir, una de las palabras más repetidas y usadas en redes sociales. Esto debido a que está siendo implementada en diversos sectores de la sociedad, tales como el sector transporte, el cual cuenta con vehículos autónomos (Falcone et al., 2007). Así también, en el sector salud, creando programas de detección de enfermedades cancerígenas, y en el sector empresarial, en el que la forma más común del uso de la IA es a través de agentes conversacionales que permiten mejorar la relación con el cliente (Kohn et al., 2014), entre otros aportes que ayudan y benefician al desarrollo de la sociedad.

La IA ha sido empleada en las organizaciones durante mucho tiempo, debido a que permite resolver problemas y automatizar tareas (Wamba-Taguimdje et al., 2020), dando a las empresas y al área de gestión una nueva dimensión (Haefner et al., 2021). Actualmente, la IA está escalando de forma abrupta en las organizaciones, lo que genera un impacto complejo en los trabajos futuros. Este nuevo intelecto ofrece beneficios a las organizaciones, como el aumento de la productividad, la optimización de procesos, el fomento de la innovación, etcétera. (Mayer et al., 2020), volviéndose más eficientes y competitivas en el mercado.

Las organizaciones ven este nuevo avance como un medio para llegar a su finalidad o visión como empresa. En relación con eso, la IA ha convertido este proceso en uno más inteligente, optimizado, competente y mecánico, permitiendo eliminar procesos deficientes que se ejecutaban de forma manual y requerían de recursos significativos (Wamba-Taguimdje et al., 2020).

Se deben distinguir dos efectos de la implementación de la IA: la automatización y la complementariedad (Raisch y Krakowski, 2020). La primera implica que la máquina asume el rol del ser humano, encargándose de tareas que a este le corresponden; mientras que la segunda, significa que tanto el hombre como la máquina realizan la tarea, es decir, que la 
máquina sirve de apoyo para que el hombre pueda realizar tareas de una forma más eficiente y eficaz.

En los últimos años, se está dando una rápida visibilidad de impulso e implementación de inteligencia artificial, como por ejemplo el caso de Watson de IBM: un programa informático de IA, diseñado para responder preguntas del lenguaje natural en una variedad de actividades del mundo real (marketing, administración, justicia, atención médica) (Kohn et al., 2014). Precisamente, el procesamiento del lenguaje natural le da la capacidad de comprender oraciones compuestas por humanos con matrices y asignar múltiples significados o conceptos. Las capacidades de aprendizaje automático permiten a Watson aprender de la experiencia y la interacción con los datos desarrollando soluciones inteligentes basadas en experiencias (Jarrahi, 2018). Los sistemas de IA emergentes como Watson poseen una gran capacidad para aprender y mejorarse a sí mismos, acelerando su uso para algunas tareas basadas en el conocimiento.

Otro ejemplo de uso de la IA es el caso de la Compañía petrolera nacional de Abu Dhabi (ADNOC) es un importante grupo diversificado de empresas energéticas y petroquímicas en Abu Dhabi. Esta empresa ha adoptado la IA, mediante la tecnología de IBM, con el fin de garantizar la automatización de sus diferentes operaciones y procesos, incluido el análisis y categorización de muestras de rocas, acelerando así el desarrollo de modelos geológicos digitales de yacimientos (Kohn et al., 2014).

\subsection{Toma de decisiones y decisiones gerenciales}

De acuerdo con Pomerol (1997, pág. 3), la toma de decisiones se puede considerar sencillamente como «elegir entre varias alternativas». Siguiendo esta lógica, se podría decir que esto cubriría todas las aplicaciones de la IA, como es el caso del reconocimiento de imágenes o la comprensión del lenguaje natural, la cual se basa en una elección entre un gran número de sujetos u oraciones. Sin embargo, lo mejor es restringir las consideraciones a situaciones que comienzan con el reconocimiento, de que es necesario y esencial tomar una decisión, esto conduce a alguna forma de diagnóstico o diseño, seguido de un proceso de elección y finalmente la acción (Cao et al., 2021).

Llevado esto a un plano organizacional, se puede decir que la toma de decisiones gerenciales consiste en identificar una problemática hasta darle solución, siguiendo un proceso que comprende obtener información, luego tomar una decisión, llevarla a la acción, para finalmente visualizarlo en el desempeño de la empresa. Pero en la realidad este proceso no es sencillo, debido a que el tomador de decisiones encuentra una serie de obstáculos como la incertidumbre, la cual resulta un elemento con un fuerte impacto al momento de tomar decisiones. De igual forma, otro inconveniente suele ser la falta de información sobre todas 
las alternativas o sus posibles consecuencias, lo que dificulta la interpretación de una situación y la toma de una decisión (Choo, 1991, como se citó en Jarrahi, 2018).

\subsection{Relación de la IA con la toma de decisiones gerenciales}

Según Cao (2021), existieron dos épocas principales relacionadas al uso de la inteligencia artificial con la toma de decisiones gerenciales. La primera época comenzó a partir de la 1970, y alcanzó su apogeo en 1990. La segunda época todavía no ha concluido, es la que se está viviendo actualmente. Desde inicios de 1980, especialistas e investigadores tomaron en cuenta la importancia de invertir en tecnología y en el desempeño organizacional (Farhanghi et al., 2013; Nwamen, 2006; Ruiz-Mercader et al., 2006). A causa de ello, la inteligencia artificial logró avances concretos en los años 2000 con el «aprendizaje automático» (Yoav Shoham, 2018).

Más adelante, se adoptó una visión de contingencia, que consistía en separar las actividades, dejando a las máquinas realizar las operaciones de rutina y a los humanos las actividades administrativas complejas. Esto produjo que la inteligencia artificial se centrará en la computación y en los aspectos operativos, mientras que los humanos en la gestión (Rahwan et al., 2019; Simon, 1987).

En la actualidad, existen muchas investigaciones en torno al uso de la inteligencia artificial con el objetivo de mejorar y optimizar los procesos (P. Burggräf, J. Wagner, B. Koke, et al., 2020). Ahora, las máquinas ya no solo realizan actividades mecánicas, sino que también están aptas para desempeñar trabajos cognitivos. Cabe mencionar que las máquinas aún poseen muchas limitaciones, por lo que existe una relación hombre-máquina. Este nuevo enfoque permite la complementariedad de las capacidades de las máquinas y de los seres humanos (Daugherty y Wilson, 2018).

La IA y otras tecnologías inteligentes pueden ayudar a los tomadores de decisiones humanos con análisis predictivos, generando ideas frescas a través de enfoques de inferencia estadística, basada en datos y probabilidades, como también identificar relaciones entre los diferentes factores, lo que permite que estos puedan recopilar de manera más efectiva y actuar sobre nuevos conjuntos de información. Una de las funciones principales del análisis predictivo es generar nueva información y predicciones sobre clientes, activos y operaciones. Una encuesta reciente por Accenture (2017), reveló que el 85 \% de los encuestados ejecutivos tienen planes de invertir ampliamente en tecnologías relacionadas con la IA durante los próximos 3 años (Jarrahi, 2018).

Las organizaciones siempre han aspirado a asignar más tareas a las máquinas con el fin de reducir costos y mejorar la eficiencia. Todo esto comenzó con las líneas de montaje, que reemplazaron el trabajo humano en tareas mecánicas y repetitivas antes conocidas como 
«labor manual» (Dopico et al., 2016). Dados los rápidos avances tecnológicos, particularmente en el campo de la IA, la idea de confiar tareas más complejas a las máquinas ya no parece tan descabellada como lo era hace varios años, debido a las diferentes capacidades y configuraciones que poseen las diferentes tecnologías de información, lo cual ha demostrado su eficacia en la automatización de tareas monótonas y repetitivas, generalmente realizadas por especialistas como administradores de recursos humanos, vendedores y pequeños contratistas (Rachinger et al., 2019).

En este sentido, la IA y otras tecnologías inteligentes a menudo se discuten como el epicentro de una ola de automatización sin precedentes. Además, se les considera como impulsores de la transformación, debido a que poseen procesos cognitivos complejos para la toma de decisiones (MacCrory et al., 2014).

\subsubsection{Decisiones intuitivas como ventaja humana.}

Muchas veces las empresas se enfrentan a situaciones críticas donde se requieren decisiones rápidas, por lo cual se necesitará de la intuición humana para escoger soluciones acertadas en estos momentos de incertidumbre (Jarrahi, 2018). Según Frantz (2003), la intuición consiste en reconocer los patrones subconscientes, y se considera como un efecto colateral positivo producto de un largo periodo de estudio. Además, forma parte del procesamiento de la información humana.

Las tecnologías basadas en la IA analizan contextos de decisión generalmente fundados en probabilidades (Guszcza et al., 2017), como también, en sistemas racionales y analíticos, pero estas podrían estar mal equipadas para abordar problemas y situaciones novedosas. Es así que el pensamiento analítico y probabilístico en la toma de decisiones se torna insuficiente aterrizados en la perpleja vida real (Dane y Pratt, 2007). Presentado este contexto, los tomadores de decisiones humanos, generalmente, se basan en un enfoque intuitivo, aprovechando el conocimiento, la experiencia supuesta, las emociones y el juicio personal (Jarrahi, 2018). También se dice que la intuición puede llegar a ser fundamental para poder completar con éxito diversas tareas que involucran una alta complejidad en periodos cortos de tiempo, como la planificación corporativa, el análisis de acciones y la evaluación del desempeño (Dane y Pratt, 2007).

Por ejemplo, Apple, al momento de tomar sus decisiones, no suele considerar estudios, encuestas o investigaciones extensas, por lo que es inusual que las decisiones importantes requieran varios meses. Steve Jobs, el fundador, justamente se hizo conocido por tomar decisiones rápidas, pero intuitivas y esto se evidencia en el caso de los primeros iMacs, cuando Jobs decidió de manera inmediata que Apple debería lanzar las nuevas computadoras en un arco iris de colores dulces. Jony Ive, director de diseño de Apple, señaló: 
«En la mayoría de los lugares, esa decisión habría llevado meses, Steve lo hizo en media hora» (Isaacson y Scanziani, 2011, pg.356). Entonces se observa que el ingenio de Jobs no radica necesariamente en comprender las probabilidades de éxito, sino en dar soluciones que parecían holísticamente sensatas basadas en el instinto.

\subsubsection{Ventaja de la IA en la simplificación de la toma de decisiones.}

Existen varios factores que pueden influir en la decisión de optar por la IA, uno de ellos consiste en que los gerentes se enfrentan a entornos cada vez más competitivos y cambiantes, mercados internacionales cada vez más exigentes y difíciles de penetrar, además de posibles panoramas políticos inestables. En comparación con la gestión efectuada por el ser humano, la efectuada por la IA puede provocar un cambio disruptivo, y surge la duda de saber hasta qué punto la IA puede reemplazar a los seres humanos (Haefner et al., 2021); más, sin embargo, lo que sería visto como una desventaja para muchos trabajadores, podría también significar una oportunidad.

Se debe considerar que la finalidad de la toma de decisiones está ligada al hallazgo continuo de mejores soluciones para el beneficio empresarial, por ello también debemos tener en cuenta el posible acoplamiento de la IA en la toma de decisiones gerenciales, por las ventajas que puede proveer a las organizaciones. Es así que, en el ámbito de la gestión empresarial, la IA se utiliza como una herramienta que brinda ventajas competitivas frente a otras organizaciones; ya que aporta mayor calidad y eficiencia en la toma de decisiones en comparación a los seres humanos, y de esta manera puede brindar mejores resultados en la organización (Haefner et al., 2021).

La IA expresa su potencial por medio de la detección y la predicción de los comportamientos humanos (Wamba-Taguimdje et al., 2020). Teniendo esto en cuenta, es preciso relacionar la toma de decisiones gerenciales con la innovación empresarial. Como tecnología innovadora y a menudo cambiante, los efectos transformadores de la IA se materializan en los estudios de casos sobre el rediseño de las estructuras organizacionales por medio de la descentralización de la toma de decisiones básicas por parte del personal. De este modo, contribuyen a un mejor desempeño financiero, comercial y administrativo (WambaTaguimdje et al., 2020).

Al presentarse situaciones en las que se requiera una gran capacidad de procesamiento de información, la IA toma este papel superando a la capacidad humana, en lo analítico, cuantitativo y computacional. Así, la IA al poseer la ventaja de la fuerza bruta, la convierte en una herramienta rigurosa y eficiente para recuperar y analizar grandes cantidades de datos, mejorando la complejidad del dominio de un problema. Por ende, la IA al tener grandes capacidades contribuye en diferentes ámbitos, como evaluar el riesgo 
crediticio de una persona mediante el examen de su lista de amigos en Facebook, los anuncios de precios en marketing digital y la suscripción de hipotecas en la industria inmobiliaria de EE. UU., y con el pasar de los años se ha logrado que la máquina aprenda de los datos sin procesar y se expanda integrando conjuntos de datos más grandes, así estas ofrecen calidad en la toma de decisiones (Jarrahi, 2018).

\section{Metodología}

El presente trabajo de investigación empleó como metodología una «Revisión Sistemática de la Literatura» o como se la conoce en inglés Systematic Literature Review (SLR). Según Tranfield et al. (2003), la revisión sistemática permite hallar e identificar contribuciones científicas fundamentales en un área determinada. Este método plantea seguir una serie de etapas para una correcta revisión; primero, enunciar la pregunta de investigación y ubicar la literatura; segundo, seleccionar y estimar la información encontrada; tercero, analizar y sintetizar la literatura; y por último, exhibir y utilizar los resultados. De esta manera se establece una revisión de la literatura ordenada y rigurosa. Agregando a lo anterior, se empleó el método inductivo haciendo uso de colores para una adecuada recopilación de información, y así armar una ordenada estructura del trabajo.

Por otra parte, se revisaron artículos pertenecientes a revistas clasificadas en el cuartil $1(\mathrm{Q} 1)$ y cuartil 2 (Q2), lo cual muestra la importancia relativa de estas dentro del total de revistas de su área. Se seleccionaron artículos principalmente en el idioma inglés, se descartaron tesis, simposios, actas de congresos, libros y otros trabajos similares. Se recurrió a bases de datos como Scopus y ScienceDirect por la calidad validada y la confiabilidad de las investigaciones que estos motores de búsqueda presentan. A las cuales se accedió empleando el servicio de biblioteca electrónica que facilita la Universidad Nacional Mayor de San Marcos.

Realizada la selección de bases de datos confiables y de calidad, se procedió a aplicar una estrategia de búsqueda avanzada apoyada en la selección de palabras clave o keywords, que son, según González y Mattar (2012), términos o frases cortas (lexemas) que permiten clasificar y direccionar las entradas en los sistemas de indexación y de recuperación de la información en las bases de datos de un manuscrito o área temática en particular. Estas fueron «inteligencia artificial», «decisiones gerenciales» y sus términos relacionados en cuanto a sinónimos y traducciones en inglés. Para generar estos canales de búsqueda avanzada, se utilizaron operadores booleanos (OR, AND), operadores de proximidad $(\mathrm{W} / \mathrm{n})$ y operadores de texto ("', \{\}$)$.

Los artículos usados son recientes, ya que según el Al Index Report de 2019, la cantidad de artículos acerca de la IA ha tenido un crecimiento exponencial en la presente 
década. Estos pertenecen al área de Gestión organizacional, Finanzas, Negocios, Ciencias de la decisión, Economía y Contabilidad.

\section{Tabla 1}

Resumen de la metodología empleada.

\begin{tabular}{|c|c|c|}
\hline $\begin{array}{l}\text { Objetivos de } \\
\text { investigación }\end{array}$ & \multicolumn{2}{|c|}{$\begin{array}{l}\text { - Analizar los conceptos de Inteligencia Artificial y Toma de } \\
\text { decisiones, y su relación en el mundo de los negocios. } \\
\text { - Identificar la influencia que conlleva esta interrelación. }\end{array}$} \\
\hline & Inteligencia artificial & Decisiones gerenciales \\
\hline $\begin{array}{l}\text { Palabras clave } \\
\text { empleadas en la } \\
\text { búsqueda }\end{array}$ & $\begin{array}{l}\text { inteligencia artificial, artificial } \\
\text { intelligence, computación } \\
\text { cognitiva, computación } \\
\text { autónoma, aprendizaje } \\
\text { automático, machine } \\
\text { learning, cognitive } \\
\text { computing. }\end{array}$ & $\begin{array}{l}\text { decisiones administrativas, } \\
\text { planeamiento estratégico, } \\
\text { managerial decisions, } \\
\text { administrative decisions, } \\
\text { administrative planning, } \\
\text { strategic planning, decision } \\
\text { making. }\end{array}$ \\
\hline Motores de búsqueda & Scopus & Science Direct \\
\hline Criterio de selección & \multicolumn{2}{|c|}{$\begin{array}{l}\text { Proceso formado por las } 5 \text { etapas empleadas en la } \\
\text { metodología SLR }\end{array}$} \\
\hline Análisis y síntesis & $\begin{array}{l}\text { Enfoque Cualitativo } \\
\text { Método Inductivo }\end{array}$ & \\
\hline
\end{tabular}

Fuente: Elaboración propia.

\section{Tabla 2}

Clasificación de las revistas usadas según cuartiles.

\begin{tabular}{lc}
\hline \multicolumn{1}{c}{ Revistas } & Cuartiles \\
\hline Technovation & Q1 \\
Academy of management review & Q1 \\
Business Research & Q2 \\
Business horizons & Q1 \\
Journal of business and psychology & Q1 \\
\hline
\end{tabular}


$\begin{array}{ll}\text { Management Review Quarterly } & \text { Q1 }\end{array}$

$\begin{array}{ll}\text { MIT Sloan Management Review } & \text { Q1 }\end{array}$

$\begin{array}{ll}\text { European Journal of Operational Research } & \text { Q1 }\end{array}$

$\begin{array}{ll}\text { California Management Review } & \text { Q1 }\end{array}$

Business Process Management Journal $\quad$ Q1

$\begin{array}{ll}\text { Technological Forecasting and Social Change } & \text { Q1 }\end{array}$

$\begin{array}{ll}\text { Journal of Economic Psychology } & \text { Q1 }\end{array}$

$\begin{array}{ll}\text { Industrial Marketing Management } & \text { Q1 }\end{array}$

$\begin{array}{ll}\text { Procedia CIRP } & \text { Q2 }\end{array}$

Foundations and Trends in Entrepreneurship $\quad$ Q1

$\begin{array}{ll}\text { Decision Support Systems } & \text { Q1 }\end{array}$

$\begin{array}{ll}\text { MIS Quarterly Executive } & \text { Q1 }\end{array}$

Journal of Personality and Social Psychology $\quad$ Q1

$\begin{array}{ll}\text { Personality and Social Psychology Review } & \text { Q1 }\end{array}$

Fuente: Elaboración propia.

\section{Resultados de la revisión}

Los resultados de este estudio, tras el análisis de la información, se centran en la fuerte dependencia de la toma de decisiones hacia la IA, debido a la necesidad que tienen las organizaciones de mejorar su proceso, valor comercial, imagen, entre otros aspectos.

A partir de la información analizada, queda evidenciado que la IA y la tecnología que ofrece son herramientas relevantes para mejorar el desempeño de la organización, debido a que se pueden eliminar procesos innecesarios, reducir tiempos y costos; y, por lo tanto, mejorar la eficiencia de la empresa, adaptándola a los diferentes desafíos que se vienen suscitando en cuanto a competitividad.

Además, se ha podido constatar que el proceso de toma de decisiones en una empresa es un factor decisivo para el futuro de esta. Por ello, la disyuntiva de quién debería realizar esta fundamental tarea organizacional, ya sea el hombre o la IA, es una elección que 
no debe tomarse a la ligera. Asimismo, poder decidir entre ambas opciones es una labor un tanto complicada, pues ambas presentan, individualmente, ventajas muy tentadoras.

Por su parte, el ser humano se apoya en la intuición al momento de tomar decisiones, una característica que resulta ser inherente a su procesamiento de información y que claramente la IA no puede igualar. Por otro lado, la IA puede ofrecer a la gerencia, dentro de la toma de decisiones, una ventaja competitiva por encima del ser humano, en lo que respecta a un rápido procesamiento y eficiente análisis de grandes cantidades de datos, simplificando de esta manera la toma de decisiones. Sin embargo, esto no quiere decir que la IA busca ser un reemplazo inminente del ser humano, sino por el contrario, su principal finalidad es complementar y expandir la habilidad humana, en lugar de restringirla o disminuirla.

El estudio «Big data and Al Executive Survey 2019», elaborado por NewVantage Partners, en el que se realizó una encuesta a 60 empresas de la lista Fortune 1000, concluyó que $76.5 \%$ de los ejecutivos consideraban que el big data o las grandes cantidades de datos, y la inteligencia artificial se están entrelazando; pues cada vez aumenta la información que está al alcance de las empresas, es por ello que se vuelve imprescindible la IA en las organizaciones para poder encontrar patrones y darle sentido a los datos disponibles. De igual manera, el $97.2 \%$ de las compañías encuestadas está invirtiendo en Big Data e iniciativas de IA, con la finalidad de que puedan evolucionar y convertirse en empresas ágiles y basadas en datos. Y el 91.6 \% está aumentando su ritmo de inversión en Big Data e IA.

Es así que queda demostrado que la IA será, en los posteriores años, una herramienta muy útil o necesaria, y por qué no, tal vez hasta indispensable para que una empresa llegue a ser exitosa.

\section{Discusión}

De acuerdo con los aportes teóricos que se presentaron anteriormente, se infiere que uno de los temas que genera discusión es la automatización, debido a que, según WambaTaguimdje et al. (2020), en los últimos años se asigna y confía a las máquinas tareas más complejas, asimismo la IA se vuelve cada vez más omnipresente en los contextos de gestión.

En este sentido, la IA toma un papel fundamental, ya que el gerente encargado se va a encontrar frente a una decisión importante: o seguir con la forma tradicional, en donde los seres humanos son lo que deciden y se hace uso de los métodos ya conocidos, o decide hacer uso de la IA para la toma de decisiones. La primera opción parece ser la más confiable por ser la más usada, adicionalmente los seres humanos tienen la capacidad de actuar rápidamente frente a situaciones sin precedentes, algo que no podrían hacer las máquinas. Por otro lado, utilizar la IA en la toma de decisiones es algo novedoso, puesto que puede 
ofrecer mejores resultados en el ámbito de la gestión al ser las mismas máquinas las programadas para desempeñar esta tarea.

Se considera que en un punto la automatización en la toma de decisiones se vuelve un problema cuando se busca desplazar integralmente al hombre, lo que conllevaría a diversas consecuencias. Tales como limitar la capacidad de los tomadores de decisiones, ya que estos deben regirse a lo que la máquina predispone. Así como lo menciona Skitka et al. (2000) esto genera la disminución de las capacidades, puesto que vuelve a los tomadores de decisiones complacientes e irresponsables, generando pérdidas de las habilidades humanas en las empresas (Endsley y Kiris, 1995).

Otra de las consecuencias que se considera que se suscitan por esta automatización es la diferenciación de información que manejan los que están adaptados a esta digitalización con los que carecen de conocimientos tecnológicos, lo que genera tensiones cuando hay cambios en el tiempo (Smith y Lewis, 2011), estos cambios importantes hacen que las soluciones automatizadas sean menos eficientes (Davenport y Kirby, 2016), lo que genera que algunos se logran adaptar y otros quedan desfasados ante esta velocidad de la transformación digital.

Existen varios ejemplos donde se refleja que el uso de la IA de manera desmesurada puede conllevar a errores en la toma de decisiones. Tales como podría ser la implicación de la tecnología en los procesos de elección de personas que puede conllevar a problemas sociales. Como indica Daughtrty y Wilson (2018) estos problemas sociales en la toma de decisiones se pueden visualizar en las entidades financieras al momento de otorgar un crédito bancario, en muchas situaciones estas decisiones se realizan por procesos automatizados que dan elegibilidad crediticia por origen étnico, género o código postal, que en diversos casos poseen sesgos de la máquina causados por datos ruidosos, errores estadísticos y / o predicciones socialmente molestos, que a menudo conducen a una discriminación nueva y más sistemática (Raisch y Krakowsk, 2020). Además de esto, las organizaciones con toma de decisiones totalmente enfocadas a la automatización pueden ser objeto de manipulación, debido a la ética de diseño de los ingenieros informáticos que usan esto para su beneficio empresarial.

Es así que se sostiene la idea de que para una buena toma de decisiones es necesario el uso de la inteligencia artificial, con una respectiva supervisión, ya que, como lo afirman (Davenport y Kirby, 2016: 21), esto podría otorgar resultados mejor analizados y menos propensos a errores.

Se tomó la postura, entonces, de que la intuición del ser humano y la gran capacidad analítica de la inteligencia artificial no deberían operar de manera independiente, la toma de 
decisiones organizacionales se llevaría de mejor manera combinando estos dos enfoques, debido a que se complementan entre sí. Como afirma Martin (2009. p.6), los aspectos del pensamiento tanto analítico como intuitivo, de forma aislada, son necesarios, pero no suficientes para un rendimiento empresarial óptimo, de hecho, las empresas más exitosas en los próximos años equilibrarán el dominio analítico y la originalidad intuitiva.

Ante ello, se estima que la intuición no puede funcionar, a menos que haya datos disponibles para que el ser humano pueda procesar y combinar con la experiencia pasada (como motor de la intuición) junto con el análisis basado en datos. Esto se respalda con la postura anteriormente planteada por Simon, que no considera que la intuición fuera irracional, al contrario, la consideraba un método analítico racional, pero no consciente de toma de decisiones.

Hay varios ejemplos de esta visión compartida. Reid Hoffman, presidente ejecutivo de Linkedln, dijo que los sistemas de inteligencia artificial permiten a los humanos tomar mejores decisiones, ya que estos «pueden examinar grandes cantidades de datos para resaltar las cosas más interesantes, en cuyo punto los gerentes pueden profundizar, utilizando la inteligencia humana, para llegar a conclusiones y tomar acciones» (Hoffman, 2016). De igual forma, los bots ahora detectan contenido inapropiado o controvertido en la web o en las redes sociales al analizar y procesar terabytes de datos generados por el usuario, pero la decisión final de eliminar publicaciones o videos en las redes sociales a menudo recae en los trabajadores «detrás de la cortina de la inteligencia artificial» que usa un juicio humano superior (Gray y Suri, 2017). Esto ayuda a identificar qué factores deben estar en primer plano en la recopilación y el análisis de datos, que será la tarea principal de las tecnologías inteligentes.

\section{Conclusiones}

De acuerdo con los temas desarrollados, se concluye que en la actualidad la inteligencia artificial es un instrumento que mejora y optimiza los procesos existentes, del mismo modo su uso se está incrementando y popularizando en las empresas. Asimismo, la IA ayuda y facilita las elecciones en la toma de decisiones por medio de la creación de patrones establecidos frente a circunstancias o eventos que requieren decisiones.

Sin embargo, es importante mencionar que la Inteligencia Artificial sirve de ayuda, únicamente, frente a contextos ya conocidos, evaluados y analizados previamente; por lo que frente a contextos que surgen súbitamente, la mejor opción es que el gerente encargado use su ventaja intuitiva para escoger de la mejor manera posible una solución. Asimismo, se espera que en un futuro la IA logre desarrollar un mecanismo de anticipación frente a nuevas 
y más complejas situaciones, sólo así la IA podría actuar de forma independiente en la toma de decisiones.

Por ende, se concluye que, en la actualidad, la IA debe ser usada como una herramienta de apoyo y complemento en la toma de decisiones, y su uso debe ser supervisado y evaluado constantemente por un personal capacitado para dicha función, y así se tomen mejores decisiones en la empresa del contexto actual, el cual está en constante cambio.

\section{Referencias}

Aaldering, L. y Song, C. (2020). Of leaders and laggards - Towards digitalization of the process industries, Technovation, 105 102312. https://doi.org/10.1016/j.technovation.2020.102211

Ahituv, N., Igbaria, M., and Sella, A. (1998). The Effects of Time Pressure and Completeness of Information on Decision Making, Journal of Management Information Systems, 15 (2), 153-172. https://doi.org/10.1080/07421222.1998.11518212

Baumeister, R. (2003). The psychology of irrationality: Why people make foolish, self-defeating choices. The psychology of economic decisions, ed. Isabelle Brocas and Juan D. Carrillo, 1, 3-16. https://doi.org/10.1111/j.1745-6916.2008.00057.x

Burggräf, P., Wagner, J., Koke, B., \& Bamberg, M. (2020). Performance assessment methodology for Al-supported decision-making in production management. Procedia CIRP, 93, 891-896. https://doi.org/10.1016/J.PROCIR.2020.03.047

Cao, G., Duan, Y., Edwards, J. S., \& Dwivedi, Y. K. (2021). Understanding managers' attitudes and behavioral intentions towards using artificial intelligence for organizational decisionmaking. Technovation, 106. https://doi.org/10.1016/j.technovation.2021.102312

Dane, E., y Pratt, M. (2007). Exploring intuition and its role in managerial decision making. Academy of Management Review, 32(1), 33-54. https://doi.org/10.2307/20159279

Davenport, T. H., y Kirby, J. (2016). SPRING 2016 Just How Smart Are Smart Machines?. MIT Sloan Management Review, 1(3), 7. https://sloanreview.mit.edu/article/just-how-smartare-smart-machines/

Daugherty, P. R., y Wilson, J. H. (2018). Human + Machine: Reimagining Work in the Age of Al. Harvard Business Review Press.

De Dreu, C., Nijstad, B., y Knippenberg, D. (2008). Motivated Information Processing in Group Judgment and Decision Making. Personality and Social Psychology Review, 12 (1), 2249.

https://citeseerx.ist.psu.edu/viewdoc/download?doi=10.1.1.844.9957\&rep=rep1\&type=pd $f$ 
Dopico, M., Gomez, A., De la Fuente, D., García, N., Rosillo, R., y Puche, J. (2016). A vision of industry 4.0 from an artificial intelligence point of view. The Steering Committee of The World Congress in Computer Science, Computer Engineering and Applied Computing (WorldComp).

Endsley, M., y Kiris, E. (1995). El problema de rendimiento fuera del circuito y el nivel de control en automatización. Factores humanos, 37, 381-394. https://doi.org/10.1518/001872095779064555

Falcone, P., Borrelli, F., Asgari, J., Tseng, H. E., y Hrovat, D. (2007). Predictive active steering control for autonomous vehicle systems. IEEE Transactions on control systems technology, 15(3), 566-580. https://doi.org/10.1109/TCST.2007.894653

Farhanghi, A. A., Abbaspour, A., y Ghassemi, R. A. (2013). The Effect of Information Technology on Organizational Structure and Firm Performance: An Analysis of Consultant Engineers Firms (CEF) in Iran. Procedia-Social and Behavioral Sciences, 81, 644-649. https://doi.org/10.1016/j.sbspro.2013.06.490

Frantz, R. (2003). Herbert Simon. Artificial intelligence as a framework for understanding intuition. Journal of Economic Psychology, 24(2), 265-277. https://doi.org/10.1016/S0167-4870(02)00207-6

González, M., y Mattar, S. (2012). Las claves de las palabras clave en los artículos científicos. Revista MVZ Córdoba, 17(2), 2955-2956. https://www.redalyc.org/articulo.oa?id=69323751001

Guszcza, J., Evans-Greenwood, P., y Lewis, H. (2017). Cognitive collaboration: Why humans $\begin{array}{llll}\text { and } & \text { computers. } & \text { Deloitte } & \text { 8-29. }\end{array}$ https://www2.deloitte.com/us/en/insights/deloitte-review/issue-20/augmentedintelligence-human-computer-collaboration.html

Haefner, N., Wincent, J., Parida, V., y Gassmann, O. (2021). Artificial intelligence and innovation management: A review, framework, and research agenda放. Technological $\begin{array}{llll}\text { Forecasting } \text { and Social } & \text { 120-392. }\end{array}$ https://doi.org/10.1016/J.TECHFORE.2020.120392

Isaacson, J. S., y Scanziani, M. (2011). How Inhibition Shapes Cortical Activity. Neuron, 72(2), 231-243. https://doi.org/10.1016/J.NEURON.2011.09.027

Janis, I. (2008). Groupthink, IEEE Engineering Management Review, 36 (1), 36-36. https://doi.org/10.1109/EMR.2008.4490137

Jarrahi, M. H. (2018). Artificial intelligence and the future of work: Human-Al symbiosis in organizational decision making. Business Horizons, 61(4), 577-586. https://doi.org/10.1016/j.bushor.2018.03.007 
Kohn, N., Eickhoff, S. B., Scheller, M., Laird, A. R., Fox, P. T., y Habel, U. (2014). Neural network of cognitive emotion regulation - An ALE meta-analysis and MACM analysis. Neurolmage, 87, 345-355. https://doi.org/10.1016/J.NEUROIMAGE.2013.11.001

Lyengar, S. S., y Lepper, M. R. (2000). When choice is demotivating: Can one desire too much of a good thing? Journal of Personality and Social Psychology, 79(6), 995-1006. https://faculty.washington.edu/jdb/345/345\%20Articles/lyengar\%20\%26\%20Lepper\%20( 2000).pdf

Maccrory, F., Westerman, G., Alhammadi, Y., y Brynjolfsson, E. (2014). Racing With and Against the Machine: Changes in Occupational Skill Composition in an Era of Rapid Technological Advance. Thirty-fifth International Conference on Information Systems. http://www.k12accountability.org/resources/For-

Parents/Racing_With_and_Against_the_Machine_-Changes_in_Occupational_Skill.pdf

Mayer, A. S., Strich, F., y Fiedler, M. (2020). Unintended Consequences of Introducing AI Systems for Decision Making. MIS Quarterly Executive, 19(4), 239-257. https://doi.org/10.17705/2msqe.00036

Nietzsche, R. (2015). Decision theory: How people decide and how they should decide. $\left(10^{\text {th }}\right.$ ed). Verlagshaus Mainz Gmbh, Aachen.

Nilsson, N. J. (1971). Problem-Solving Methods in Artificial Intelligence. McGraw-Hill book company. https://cse.buffalo.edu/ rapaport/572/S02/nilsson.8puzzle.pdf

Nwamen, F. (2006). Impact des technologies de l'information et de la communication sur la performance commerciale des entreprises. La Revue des Sciences de Gestion, 2, 111121. https://doi.org/10.3917/rsg.218.0111

Pomerol, J.C. (1997). Artificial Intelligence and human decision making. European Journal of Operational research, 99(1), 3-27. https://doi.org/10.1145/2987491.2987493

Rachinger, M., Rauter, R., Müller, C., Vorraber, W., y Schirgi, E. (2019). Digitalization and its influence on business model innovation. Journal of Manufacturing Technology Management, 30(8), 1741-1779. https://doi.org/10.1108/JMTM-01-2018-0020

Rahwan, I., Cebrian, M., Obradovich, N., Bongard, J., Bonnefon, J.-F., Breazeal, C., Crandall, J. W., Christakis, N. A., Mislove, A., Parkes, D. C., Sandy' Pentland, A.', Roberts, M. E., Shariff, A., Tenenbaum, J. B., y Wellman, M. (n.d.). Machine behaviour. Nicholas $R$. Jennings, 15, 26. https://doi.org/10.1038/s41586-019-1138-y

Raisch, S., y Krakowski, S. (2021). Artificial Intelligence and Management: The AutomationAugmentation Paradox. Academy of Management Review, 46(1), 192-210. https://doi.org/10.5465/amr.2018.0072

Ransbotham, S., Gerbert, P., Reeves, M., Kiron, D. y Spira, M. (2018). Artificial intelligence in business gets real, MIT Sloan Management Review and Boston Consulting Group. https://sloanreview.mit.edu/projects/artificial-intelligence-in-business-gets-real/ 
Ruiz-Mercader, J., Meroño-Cerdan, A. L., y Sabater-Sánchez, R. (2006). Information technology and learning: Their relationship and impact on organisational performance in small businesses. International Journal of Information Management, 26(1), 16-29. https://doi.org/10.1016/J.IJINFOMGT.2005.10.003

Russell, S. J., Norvig, P., Canny, J. F., Malik, J. M., y Edwards, D. D. (1995). Artificial Intelligence A Modern Approach. Prentice-Hall, Inc. https://www.cin.ufpe.br/ tfl2/artificialintelligence-modern-approach.9780131038059.25368.pdf

Simon, H. A., Dantzig, G. B., Hogarth, R., Plott, C. R., Raiffa, H., Schelling, T. C., Shepsle, K. A., Thaler, R., Tversky, A., y Winter, S. (1987). Decision Making and Problem Solving. Informs Journal on Applied Analytics, 17(5), 11-31. https://doi.org/10.1287/inte.17.5.11

Skitka, L. J., Mosier, K., y Burdick, M. D. (2000). Accountability and automation bias. International Journal of Human-Computer Studies, 52(4), 701-717. https://doi.org/10.1006/lJHC.1999.0349

Smith, WK y Lewis, MW 2011. Hacia una teoría de la paradoja: un equilibrio dinámico modelo de organización. Academy of Management Review, 36, 381-403.

Tranfield, D., Denyer, D., y Smart, P. (2003). Towards a Methodology for Developing EvidenceInformed Management Knowledge by Means of Systematic Review. British Journal of Management, 14(3), 207-222. https://doi.org/10.1111/1467-8551.00375

Trunk, A., Birkel, H., y Hartmann, E. (2020). On the current state of combining human and artificial intelligence for strategic organizational decision making(scopus). Business Research, 13(3), 875-919. https://doi.org/10.1007/s40685-020-00133-x

Wamba-Taguimdje, S. L., Fosso Wamba, S., Kala Kamdjoug, J. R., y Tchatchouang Wanko, C.E. (2020). Influence of artificial intelligence (AI) on firm performance: the business value of Al-based transformation projects. Business Process Management Journal, 26(7), 1893-1924. https://doi.org/10.1108/BPMJ-10-2019-0411

Von Krogh, G. (2018). Artificial intelligence in organizations: New opportunities for phenomenon-based theorizing, Academy of Management Discoveries, 4 (4), 404-409. https://doi.org/10.5465/amd.2018.0084

Shoham, Y., Perrault, R., Brynjolfsson, E., Clark, J., Manyika, J., Niebles, J. C., y Bauer, Z. (2018). The Al index 2018 annual report. Al Index Steering Committee, Human-Centered Al Initiative. 